\title{
Influence of nonexcitable cells on spiral breakup in two-dimensional and three-dimensional excitable media
}

\author{
K. H. W. J. ten Tusscher ${ }^{1}$ and A. V. Panfilov ${ }^{1,2}$ \\ ${ }^{1}$ Department of Theoretical Biology, Utrecht University, Padualaan 8, Utrecht $3584 \mathrm{CH}$, The Netherlands \\ ${ }^{2}$ Division of Mathematics, University of Dundee, Dundee DD1 4HN, United Kingdom \\ (Received 5 August 2003; revised manuscript received 5 September 2003; published 31 December 2003)
}

\begin{abstract}
We study spiral wave dynamics in the presence of nonexcitable cells in two-dimensional (2D) and threedimensional (3D) excitable media, described by the Aliev-Panfilov model. We find that increasing the percentage of randomly distributed nonexcitable cells can prevent the breaking up of a spiral wave into a complex spatiotemporal pattern. We show that this effect is more pronounced in $2 \mathrm{D}$ than $3 \mathrm{D}$ excitable media. We explain the observed 2D vs 3D differences by a different dependence of the period and diastolic interval of the spiral wave on the percentage of nonexcitable cells in 2D and 3D excitable media.
\end{abstract}

DOI: 10.1103/PhysRevE.68.062902

PACS number(s): 87.19.Hh, 87.19.Nn, 87.18.Bb, 05.45.Pq

Many cardiac arrhythmias are characterized by rotating waves of electrical activity [1,2], which are similar to spiral waves of excitation found in a variety of nonlinear excitable media [3]. The occurrence and multiplication of spiral waves in excitable media disrupts the spatial coherence of excitation and results in chaotic behavior. One of the most important examples is electrical turbulence in the heart (ventricular fibrillation), which is the main cause of sudden cardiac death in the world.

There are several mechanisms for the onset of turbulence in excitable media. One of them is based on the alternans instability [4,5] which occurs if the medium is excited and, instead of a periodic response (with the same period as the excitation), the durations of successive pulses of excitation (action potentials) alternate (e.g., short-long-short-long etc.). There is a simple criterion for alternans instability, based on the restitution curve of the tissue, which relates the action potential duration (APD) to the diastolic interval (DI, the time between the end of the previous action potential and the start of the next one). Alternans instability occurs if the slope of the restitution curve is more than one, leading to a flip bifurcation $[4,5]$. This criterion was found from analytical studies of one-dimensional maps and was later extended to an integral-delay equation for pulse propagation on a onedimensional ring of excitable tissue [6], where the instability occurred as an infinite dimensional Hopf bifurcation. However, recent studies show that a restitution slope steeper than one does not necessarily lead to alternans instability and that memory effects and conduction velocity restitution may also play an important role [7,8].

Numerical studies of alternans instability in two dimensions showed that instability due to a steep restitution curve can cause spiral breakup: fragmentation of a single spiral wave into a spatiotemporally chaotic pattern comprising many wavelets of various sizes [9-12]. Spiral breakup due to a steep restitution curve is now one of the most actively pursued hypotheses for the occurrence of ventricular fibrillation [9-13].

Recent experimental measurements have confirmed the role of steep restitution curves in ventricular fibrillation $[14,13]$. However, the measured values of the maximal slopes of the restitution curves were remarkably close to the boundary of the onset of instability (slope one). This implies that small changes in the characteristics of cardiac tissue could be potentially crucial for promoting or preventing breakup in the heart. One important characteristic affecting wave propagation is the conductance of gap junctions, the specialized membrane structures connecting adjacent cardiac cells [15]. The number of gap junctions changes substantially during cardiovascular disease [15]. In a previous paper we showed that decreased gap junctional conductivity can suppress spiral breakup by increasing the diastolic interval of a spiral wave [16].

Another important characteristic influencing wave propagation in the heart is the presence of so-called fibrotic, nonexcitable, "dead" tissue. In healthy hearts fibrotic tissue makes up a small percentage of the tissue. However, during aging, after a myocardial infarction (heart attack) or in particular diseases (cardiac myopathies) the percentage of fibrotic tissue increases dramatically (up to 30\%-40\%). In this paper we study how the presence of fibrotic, nonexcitable tissue will affect the process of spiral breakup in excitable media. In addition, we study in detail how the effect of nonexcitable cells differs between two-dimensional (2D) and three-dimensional (3D) excitable media. Note that because nonexcitable cells are just obstacles (heterogeneities) for propagating waves, the problem which we study here is relevant for other types of excitable media with heterogeneities, for example, the Belousov-Zhabotinsky system [17].

\section{MODEL AND RESULTS}

Excitable cells are described by the Aliev-Panfilov model [18]:

$$
\begin{gathered}
\frac{\partial e}{\partial t}=-k e(e-a)(e-1)-e r+D \Delta_{2,3} e, \\
\frac{\partial r}{\partial t}=\left[\epsilon+\left(\mu_{1} r\right) /\left(\mu_{2}+e\right)\right][-r-k e(e-b-1)] .
\end{gathered}
$$

Here the variable $e$ stands for the transmembrane potential, $D$ is the diffusion coefficient, $\Delta_{2,3}$ is the Laplacian op- 
erator, where $\Delta_{2}=\left(\partial^{2} / \partial x^{2}\right)+\left(\partial^{2} / \partial y^{2}\right)$ and $\Delta_{3}=\left(\partial^{2} / \partial x^{2}\right)$ $+\left(\partial^{2} / \partial y^{2}\right)+\left(\partial^{2} / \partial z^{2}\right)$, and variable $r$ stands for the conductance of the slow inward current. The expression $-k e(e$ $-a)(e-1)$ in Eq. (1) determines the fast processes, such as the upstroke of the action potential. The dynamics of the recovery phase of the action potential are determined by the time course of the variable $r$, mainly by the expression [ $\epsilon$ $\left.+\left(\mu_{1} r\right) /\left(\mu_{2}+e\right)\right]$. The parameters of the model do not have a clear physiological meaning but are adjusted to reproduce key characteristics of cardiac tissue, such as the shape of the action potential, refractoriness and restitution of action potential duration. The values used in this study are: $a=0.1$, $\mu_{2}=0.3, k=8, \epsilon=0.01, b=0.1$, while parameter $\mu_{1}$ is varied. The most relevant dynamical change (for spiral wave stability) as $\mu_{1}$ is varied is the steepness of the APD restitution curve, where maximum slope increases as $\mu_{1}$ is decreased.

The 2D and 3D Laplacians were numerically approximated using standard four point and six point centered difference Euler schemes. Computations were performed using an explicit time integration scheme with a time integration step of $d t=0.02$ and space integration step of $d x=0.6$, as in a previous paper [16] (both dimensionless units). No-flux boundary conditions were imposed. In 2D a $200 \times 200$ and in $3 \mathrm{D}$ a $200 \times 200 \times 76$ grid was used. Nonexcitable cells were modeled as $1 \times 1$ obstacles with Neumann boundary conditions, similar to $[19,20]$, and were randomly distributed over the medium. The size of the obstacles, scaled on the basis of a spiral wavelength of $30 \mathrm{~mm}$, was $1 \times 1 \mathrm{~mm}^{2}$. To initiate the first spiral wave we used initial data corresponding to a broken wave front, the break being located at the middle of the excitable tissue. If a spiral persisted for 80 cycles without fragmentation we considered breakup to be absent for these parameter values.

We found that increasing the percentage of nonexcitable cells suppresses spiral breakup. A few examples of the results of these computations are shown in Fig. 1. The top row shows wave patterns in a 2D sheet of excitable tissue. In Fig. 1(a) $10 \%$ of the cells are nonexcitable and we can see a turbulent wave pattern that occurred after spiral wave breakup. In Fig. 1(b) 30\% of the cells are nonexcitable, and here we can see a stable spiral wave. Similarly, the bottom row shows wave patterns in a 3D slab of excitable tissue. In Fig. 1(c) $10 \%$ of the cells are nonexcitable, again we can see a turbulent wave pattern. In Fig. 1(d) $40 \%$ of the cells are nonexcitable, and we have a stable spiral wave.

In this model spiral breakup is caused by a steep restitution curve and depends on the value of the parameter $\mu_{1}$. Decreasing of $\mu_{1}$ increases the duration and the refractory period of the action potential and increases the slope of the restitution curve. If $\mu_{1}$ is below some critical value spiral breakup occurs. Figure 2 displays the dependence of the critical value of $\mu_{1}$ on the percentage of nonexcitable cells. We see that both in 2D and 3D increasing the percentage of nonexcitable cells decreases the window in parameter space in which breakup occurs. Note also that the effect of nonexcitable cells is stronger in $2 \mathrm{D}$ sheets than in $3 \mathrm{D}$ slabs of tissue.

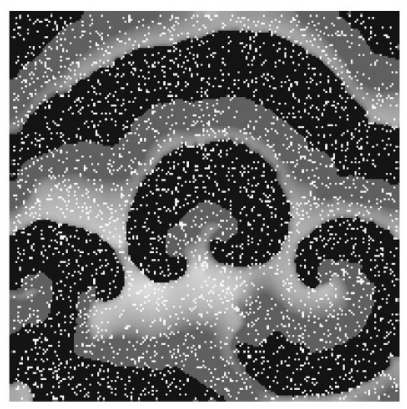

(a)

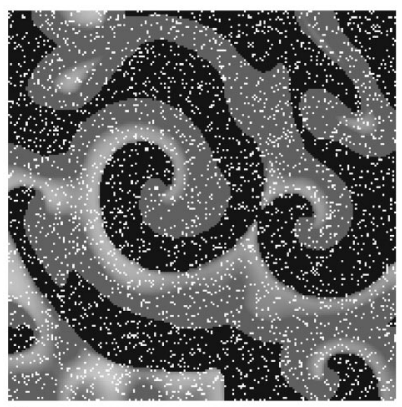

(c)

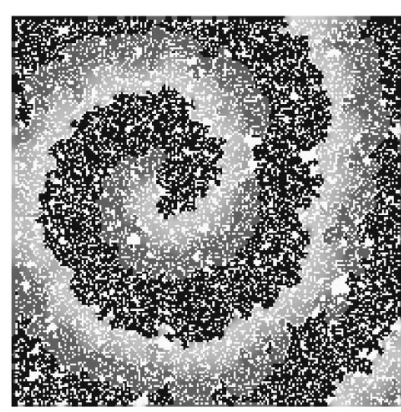

(b)

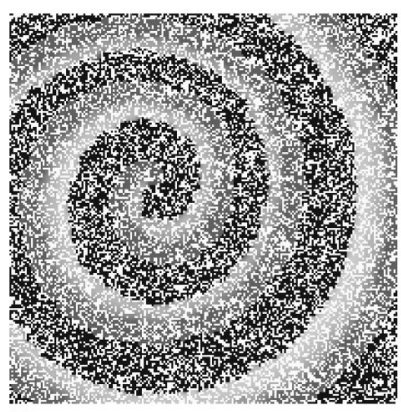

(d)
FIG. 1. (a) $2 \mathrm{D}$ wave patterns at time $t=2600$ for $\mu_{1}=0.09$ and $10 \%$ or (b) $30 \%$ of the cells being nonexcitable, (c) 3D wave patterns at time $t=2600$ for $\mu_{1}=0.11$ and $10 \%$ or (d) $40 \%$ of the cells being nonexcitable. For 3D media the wave pattern is shown in a $2 \mathrm{D}$ cross-section at the middle layer. The black area represents the excited state $(e>0.6)$ and intermediate shading from gray to white shows different levels of recovery.

To find out why breakup disappears if the percentage of nonexcitable cells increases, we studied how the main characteristics of spiral waves, such as its period and diastolic interval, are affected by the presence of nonexcitable cells (Fig. 3). For clarity, period and diastolic interval are measured for a value of $\mu_{1}=0.13$ for which no breakup occurs, as during breakup period and diastolic interval can be highly variable due to the turbulent wave pattern. From Fig. 3 it can be seen that both period and diastolic interval increase as a function of the percentage of nonexcitable cells and that this effect is much stronger in 2D sheets than in 3D slabs of tissue.

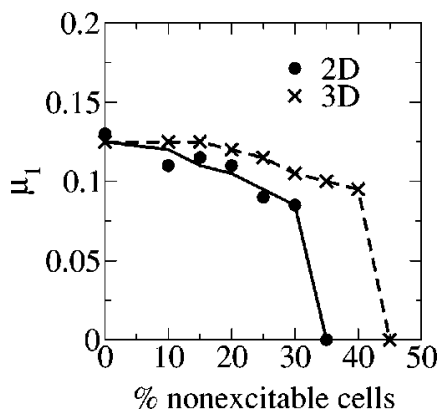

FIG. 2. Dependence of the critical value of $\mu_{1}$ below which breakup occurs on the percentage of nonexcitable cells in 2D and 3D. Results are from single simulations, however, some simulations were repeated for different patterns of nonexcitable cells and had similar outcomes (results not shown). 

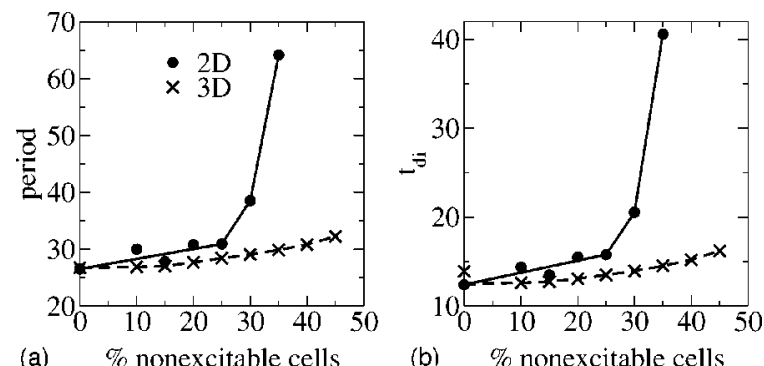

FIG. 3. (a) Average period and (b) diastolic interval $\left(t_{d i}\right)$ of a spiral wave as a function of the percentage of nonexcitable cells $\left(\mu_{1}=0.13\right)$ in $2 \mathrm{D}$ and $3 \mathrm{D}$. Results are from single simulations and for the same random patterns of nonexcitable cells as in Fig. 2.

Figure 4 illustrates how the increase in period and diastolic interval can lead to suppression of spiral breakup. The mechanism of spiral breakup in our model [Eqs. (1) and (2)] is due to a steep restitution curve [16]. Figure 4 shows such a steep restitution curve obtained for $\mu_{1}=0.09$. For illustration a line with slope one is added. On the restitution curve two points are drawn. The circle is for a $2 \mathrm{D}$ medium with $0 \%$ of the cells being nonexcitable and lies on the part with a slope steeper than one. The star is for a $2 \mathrm{D}$ medium with $30 \%$ of the cells being nonexcitable and lies on the part with a slope shallower than one. From this we can conclude that increasing the percentage of nonexcitable cells leads to an increase in diastolic interval that causes a shift along the restitution curve, away from the steep part where breakup occurs.

Because the increase of period and diastolic interval is steeper in 2D than in 3D (Fig. 3), a larger percentage of nonexcitable cells is required to suppress breakup in 3D. To explain these differences in periods, we repeated a 3D simulation for a value of $\mu_{1}=0.13$ and $30 \%$ of the cells being nonexcitable but now we decoupled the layers of the 3D slab in the $\mathrm{z}$ direction. This essentially resulted in 76 independent 2D simulations with the spatial distribution of nonexcitable cells corresponding to those of the different layers in the 3D slab. We determined the average period and diastolic interval of the spirals in each layer (Fig. 5). We see a large variability in spiral wave period between layers, with a lowest period of 29.47 (layer 32), and a highest period of 78.05 (layer 63). This suggests that due to different patterns of nonexcitable cells in the different layers the spiral waves have different

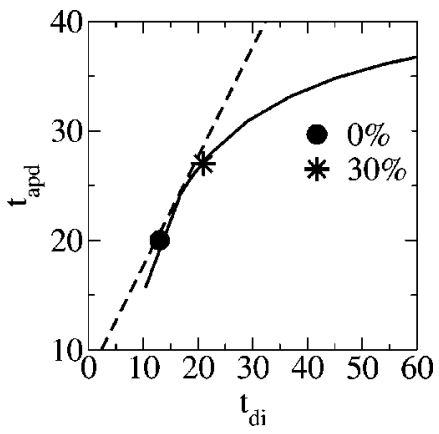

FIG. 4. Restitution curve: action potential duration $t_{\text {apd }}$ as a function of diastolic interval $t_{d i}$ for $\mu_{1}=0.09$.

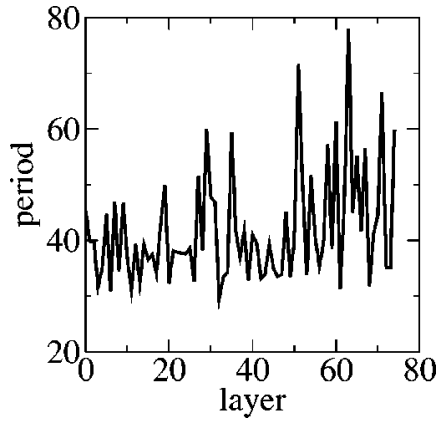

FIG. 5. Spiral wave period as a function of layer for $\mu_{1}=0.13$ and $30 \%$ of nonexcitable cells.

periods. As it was shown in [21,22], if such different layers are coupled, the 3D medium will be driven by the layer with the fastest spiral due to the electrotonic interactions between the different layers. Here we indeed see that in the completely coupled 3D simulation the scroll wave period is 29.06 , which is very close to the lowest spiral wave period recorded in the uncoupled 3D simulation.

This method of estimating the 3D period as the minimum period of the 2D layers constituting the 3D medium allows the following statistical interpretation: If $f(x)$ is the density distribution function of 2D spiral wave periods, and $F(x)$ $=\int_{-\infty}^{x} f(u) d u$ is the distribution function for the probability that a period is less than $x$, then the probability that at least one in $N$ randomly selected patterns has a period less than $x$ is $\Phi(x)=1-[1-F(x)]^{N}$. Therefore, the density distribution function for the minimum period out of $N$ periods and hence for the expected period of a 3D scroll wave in a medium with $N$ layers is $\phi(x)=d \Phi(x) / d x=N[1$ $-F(x)]^{N-1} f(x)$. Unfortunately, it is not possible to derive the density distribution function $f(x)$ analytically. Therefore, instead of using the above formulas, we decided to estimate the density distributions $f(x)$ and $\phi(x)$ by Monte Carlo simulation. For that we did 2477 simulations with different random patterns for $\mu_{1}=0.13$ and $30 \%$ of the cells being nonexcitable. Figure 6(a) shows the relative frequency distribution [estimate of $f(x)$ ] of the spiral wave periods found in these simulations. Again we see a large variability in spiral wave period: the distribution has a mean of 42.77 and a variance of 9.96. To mimic our 3D simulation consisting of 76 layers, with the fastest layer determining scroll wave period, we randomly drew 76 simulations from the total of 2477 simulations and determined the minimum period. This
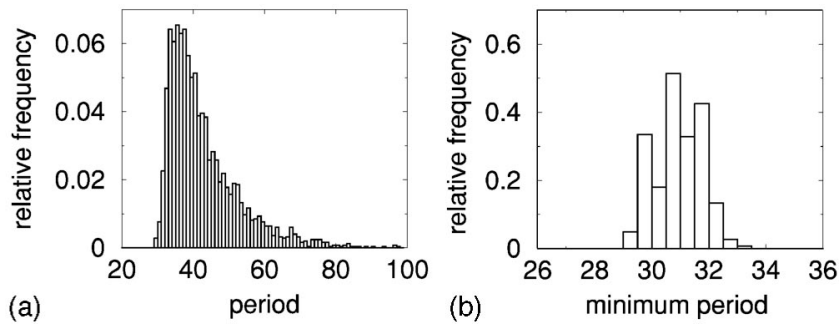

FIG. 6. (a) Relative frequency distribution of spiral wave period for 2477 simulations. (b) Relative frequency distribution of minimum period out of 76 periods for 2000 experiments. $\mu_{1}=0.13$ and $30 \%$ nonexcitable cells. 
experiment was repeated 2000 times. From this we obtained a relative frequency distribution for the minimum (out of 76) period [estimate of $\phi(x)$ ], which is shown in Fig. 6(b). The distribution has a mean of 30.93 and a variance of 0.81 . We see that the expected value for the period of a 3D scroll wave is almost $30 \%$ shorter than that of a $2 \mathrm{D}$ spiral wave. We also see that the variance of the minimum period is much smaller than that of the normal period. Note that in the case discussed in the previous paragraphs we found a period of 38.48 for the 2D spiral wave and a period of 29.06 for the 3D scroll wave, close to the mean values of the normal and minimum period, respectively [Figs. 6(a) and 6(b)]. Concluding, the difference in period between $2 \mathrm{D}$ and $3 \mathrm{D}$ media with nonexcitable cells can be explained by the fact that in 3D the scroll wave period is determined by the minimum period of multiple 2D layers, which, as follows from our statistical analysis, will on average be smaller than a single, normal $2 \mathrm{D}$ period. Note that real cardiac tissue does not consist of discrete layers. However, a "virtual" number of layers can be estimated as $N=L / l$, where $L$ is the thickness of the tissue and $l$ is the characteristic (liminal) correlation length of cardiac tissue, which is of the order of 1-2 $\mathrm{mm}$ [23].

\section{DISCUSSION}

The main result of our study is that increasing the percentage of randomly distributed nonexcitable cells suppresses restitution induced spiral breakup by causing an increase in spiral wave period and diastolic interval, leading to an upward shift along the restitution curve away from the steep part. These results are similar to results obtained in a previous study [16], where we showed a similar effect of a decreased gap junctional coupling. Together, this suggests that discontinuous conduction caused by small obstacles suppresses restitution induced spiral breakup.

In addition we showed that the effect of suppressing breakup is considerably less strong in 3D. We demonstrated that this is caused by the fact that in 3D scroll wave period is determined by the minimum period of multiple $2 \mathrm{D}$ spirals in the different layers of the 3D medium. We confirmed this finding by a statistical study.

Although our results seem to imply that fibrosis and discontinuous propagation suppress rather than promote fibrillation, drawing such a conclusion for clinical cardiology would be a gross oversimplification. There are probably many different mechanisms leading to fibrillation [24]. Spiral breakup due to a steep restitution curve is just one of the possible hypotheses. It is well known that discontinuous propagation can provoke fibrillation. Indeed, [25,26] show that decreased cell-cell coupling, heterogeneity and the presence of obstacles promotes wave break both in experiments and model simulations. Note however, that the mechanism of breakup in Refs. [25,26] is different from the steep restitution curve mechanism studied in our paper. Indeed, the model presented in Refs. $[25,26]$ has no rate dependency of APD and therefore it has a flat restitution curve. Other important differences between our simulations and the models in Refs. $[25,26]$ are the much lower excitability, the larger size of the obstacles and the absorbing as opposed to no-flux boundary conditions for the obstacles in Ref. [26]. In Ref. [26] the large objects and low excitability lead to localized conduction block, whereas in our model breakup is not site dependent and occurs as a result of dynamical instability. Summarizing, our results do not imply that fibrosis always suppresses fibrillation, but only that small nonexcitable objects in an otherwise normally excitable medium suppresses steep restitution mediated breakup.

We are grateful to Dr. J. de Bakker and Dr. E. Belitser for valuable discussions and help in this research. This research was funded by the Netherlands Organization for Scientific Research (NWO) through Grant No. 620061351 of the Research Council for Physical Sciences (EW) (K.H.W.J.T.)
[1] M.A. Allessie et al., Circ. Res. 33, 54 (1973).

[2] A.M. Pertsov et al., Circ. Res. 72, 631 (1993).

[3] A.T. Winfree et al., Nature (London) 311, 611 (1984).

[4] J. Nolasco and R. Dahlen, J. Appl. Physiol. 25, 191 (1968).

[5] M. Guevara et al., IEEE Comput. Cardiol. 562, 167 (1984).

[6] M. Courtemanche et al., Phys. Rev. Lett. 70, 2182 (1993).

[7] E.G. Tolkacheva et al., Phys. Rev. E 67, 031904 (2003).

[8] I. Banville and R.A. Gray, J. Cardiovasc. Electrophysiol. 13, 1141 (2002).

[9] A.V. Panfilov and A.V. Holden, Phys. Lett. A 147, 463 (1990).

[10] M. Courtemanche and A.T. Winfree, Int. J. Bifurcation Chaos Appl. Sci. Eng. 1, 431 (1991).

[11] H. Ito and L. Glass, Phys. Rev. Lett. 66, 671 (1991).

[12] A. Karma, Phys. Rev. Lett. 71, 1103 (1993).

[13] A. Garfinkel et al., Proc. Natl. Acad. Sci. U.S.A. 97, 6061 (2000).

[14] M. Koller et al., Am. J. Physiol. 275, H1635 (1998).

[15] H. Jongsma and R. Wilders, Circ. Res. 86, 1193 (2000).
[16] A.V. Panfilov, Phys. Rev. Lett. 88, 118101 (2002).

[17] N. Manz et al., Phys. Rev. E 66, 036207 (2002).

[18] R.R. Aliev and A.V. Panfilov, Chaos, Solitons Fractals 7, 293 (1996).

[19] A. Pertsov, in Discontinuous Conduction in the Heart, edited by P. Spooner, R. W. Joyner, and J. Jalife (Futura, Armonk, NY, 1997), Chap. 12.

[20] K.I. Agladze et al., Science 264, 1746 (1994).

[21] A.V. Panfilov, in Nonlinear wave Processes in Excitable Media, edited by A.V. Holden, M. Markus, and H.G. Othmer (Plenum Press, New York, 1991), pp. 361-382.

[22] A.V. Panfilov and J.P. Keener, Int. J. Bifurcation Chaos Appl. Sci. Eng. 3, 445 (1993).

[23] S. Weidmann, J. Physiol. (London) 118, 348 (1952).

[24] J. Jalife, Annu. Rev. Physiol. 62, 25 (2000).

[25] G. Bub, A. Shrier, and L. Glass, Phys. Rev. Lett. 88, 058101 (2002).

[26] G. Bub and A. Shrier, Chaos 12, 747 (2002). 